(c) American Dairy Science Association, 2004.

\title{
Relationships of Efficiency to Reproductive Disorders in Danish Milk Production: A Stochastic Frontier Analysis
}

\author{
L. G. Lawson, ${ }^{1,3}$ J. Bruun, ${ }^{1}$ T. Coelli, ${ }^{2}$ J. F. Agger, ${ }^{1}$ and M. Lund ${ }^{3}$ \\ ${ }^{1}$ Department of Animal Science and Animal Health, \\ The Royal Veterinary and Agricultural University, \\ Copenhagen, Denmark, \\ ${ }^{2}$ School of Economics, \\ University of Queensland, \\ Queensland, Australia, \\ ${ }^{3}$ Farm Management and Production System Division, \\ Danish Research Institute of Food Economics, \\ Copenhagen, Denmark
}

\begin{abstract}
Relationships of various reproductive disorders and milk production performance of Danish dairy farms were investigated. A stochastic frontier production function was estimated using data collected in 1998 from 514 Danish dairy farms. Measures of farm-level milk production efficiency relative to this production frontier were obtained, and relationships between milk production efficiency and the incidence risk of reproductive disorders were examined. There were moderate positive relationships between milk production efficiency and retained placenta, induction of estrus, uterine infections, ovarian cysts, and induction of birth. Inclusion of reproductive management variables showed that these moderate relationships disappeared, but directions of coefficients for almost all those variables remained the same. Dystocia showed a weak negative correlation with milk production efficiency. Farms that were mainly managed by young farmers had the highest average efficiency scores. The estimated milk losses due to inefficiency averaged 1142,488 , and $256 \mathrm{~kg}$ of energy-corrected milk per cow, respectively, for low-, medium-, and high-efficiency herds. It is concluded that the availability of younger cows, which enabled farmers to replace cows with reproductive disorders, contributed to high cow productivity in efficient farms. Thus, a high replacement rate more than compensates for the possible negative effect of reproductive disorders. The use of frontier production and efficiency/inefficiency functions to analyze herd data may enable dairy advisors to identify inefficient herds and to simulate the effect of alter-
\end{abstract}

Received February 17, 2003.

Accepted September 5, 2003.

Corresponding author: L. G. Lawson; e-mail: law@kvl.dk. native management procedures on the individual herd's efficiency.

(Key words: animal health economics, dairy efficiency, reproductive disorders, epidemiology)

Abbreviation key: DKK = Danish kroner, 1 DKK is approximately US $\$ 0.13, \mathbf{M P E}=$ milk production efficiency, SFA = stochastic frontier analysis, VE = variable expenses.

\section{INTRODUCTION}

The quantity of milk produced per cow on a farm is negatively affected by reduced reproductive performance (e.g., longer days to first-service, lower estrus detection, lower conception rates, and increased calving intervals) (Nebel and McGilliard, 1993; Fourichon et al., 2000). The level of these factors is affected by reproductive disorders. The disorders might also lead to premature culling or the disposal of production stock (Rajala-Schultz and Gröhn, 1999), which would lead to increased replacement rates. These disorders may also act as risk factors for other diseases (Erb et al., 1981; Gröhn et al., 1990) and increase the need for veterinary services. Breeding programs in several countries include selection for several attributes, such as calving ease, cow fertility traits, and disorders such as mastitis (Nielsen et al., 2000). Thus, these disorders affect farm performance in a number of ways, as measured by milk production efficiency (MPE), which is calculated as the observed milk output divided by the potential maximum milk output, given the set of input factors. Therefore, controlling reproductive disorders, such as dystocia, retained placenta, uterine infections, and ovarian cysts, could enhance the productivity and profitability of dairy herds.

Reproductive disorders have traditionally been investigated by how they affect reproduction, veterinary costs, and milk loss for an individual cow. However, the 
impact on these factors also involves farmers' complex decisions regarding culling and replacement, and the ability to ensure the optimal feeding strategy for the cows exposed to the disorders. Therefore, it is relevant to investigate impacts of reproductive diseases on production efficiency by focusing on the actions of the farmers. However, this remains to be investigated in more detail among the Danish dairy herds. This can be achieved by applying the theory of production economics (Beattie and Taylor, 1985; Debertin, 1986) and its associated estimation method, stochastic frontier analysis (SFA) (Coelli et al., 1998).

Studies in economics are generally motivated by the extent to which they affect a group of individuals-in this case, farmers operating under certain production patterns. The production pattern of dairy farms changes continually. For example, the number of dairy farms in Denmark from 1996 to 1998 decreased by $19 \%$, but herd size had increased by $17 \%$. During the same period, the average energy-corrected yields of milk per cow increased by 5\%, and labor hours used on dairy farms declined by $9 \%$ (Anonymous, 1996, 1998).

Behind these partial productivity improvements was considerable variability in milk output per cow among farms, which suggests the possibility of improvement in several farms. These changes are due to market competition among farmers and to political decisions (e.g., milk quota regulations from the European Union's Agenda 2000, which calls for no increase in milk price support and which supports soil and water environmental policies that limit the number of animals per unit of land). These factors affect farmers' decisions as herd managers.

Economic studies are formulated using theories and assumptions, and generally the theory of production states that producers (i.e., farmers) wish to maximize their profit by producing the maximal output possible given their efficiency in using the available set of input factors.

The purpose of this study is to investigate the relationship between MPE and the incidence of reproductive disorders based on reported treatments by using data from individual farms. The study, from the farmers' perspective, seeks to investigate whether farms reporting higher incidences of reproductive disorders are less efficient. Furthermore, the impact of reproductive management variables on the relationship between reproductive disorders and MPE is investigated.

\section{MATERIALS AND METHODS}

\section{Definitions}

Considering the method applied, expressions and the interpretation of results might be unfamiliar; definitions are provided to encourage easy reading. Generally, MPE measures the ability to increase milk output without increasing input factors or the ability to produce the same milk output with fewer inputs. Milk production efficiency is referred to as technical efficiency in the economic literature and is a relative measure of the productivity of input factors of any production unit that expresses its output in a quantity of any kind. Milk output elasticity is the relative percentage change in milk output due to a relative percentage change in an input factor. "First-order term" (coefficients) refers to the linear component of input factors and the "second-order term" (coefficients) refers to both the quadratic and the interaction terms. "Economic theory of production" generally assumes that farmers, in transforming their set of input factors to milk output, wish to maximize the difference between the value of milk produced and the cost of production. However, the theory also expects that the farmers are efficient in using the available set of input factors. Thus, the issue of efficiency is evaluated relative to reproductive disorders as the main focus in this study.

\section{Data}

The data used are from a stratified random sample of 514 dairy farms accounting for $4.7 \%$ of the total Danish population of 10,876 dairy farms in 1998 . The sample design took into account the region, farm area per hectare, farm economic size expressed by the gross margin, and the age of farmer. Farms were classified as dairy units if more than two-thirds of the farm's standardized gross margins came from dairy production and more than two-thirds of the dairy cattle standardized gross margins came from dairy cows. The Danish Research Institute of Food Economics provided the data on production variables. Records of treatments related to calving and the reproductive disorders and calving and culling dates for cows were collected from the National Department of Cattle Husbandry.

Danish Holstein-Friesian breed was found on 57\% of the farms; mixed breeds 20\%; Danish Jersey 13\%; and Red Danish, 10\%. Loose and tied-housing farms accounted for 30 and $70 \%$ of the sampled farms, respectively.

From 39,584 calvings in 1998, a total of 4476 cases of reproductive disorders were identified. Retained placenta accounted for most (55\%) of the disorders, followed by induction of estrus (14\%), dystocia (13\%), uterine infection (11\%), ovarian cysts (3\%), and birth induction (4\%). Reproductive disorders tended to occur at or shortly after calving. Therefore, the incidence 
risk of a particular disorder was calculated as the total number of first treatment cases reported after calving during the year divided by the total number of calvings in that year (Fourichon et al., 2001). Thus, the incidence risk is an estimate of at least one treatment per cow.

\section{The Stochastic Frontier Analysis}

To estimate MPE in Danish dairy farms, we used the SFA approach. Frontier in SFA refers to the maximal milk output for all levels of any set of input factors in a specified model for milk production. The estimated maximal potential milk output at the various levels of input factors is the frontier production curve. Stochastic in SFA refers to the random component, which is separated from the inefficiency component of the error term in statistical models in economics (econometric models). Aigner et al. (1977) and Meeusen and van den Broeck (1977) first proposed this approach, and Jondrow et al. (1982) first implemented the decomposition of the well-known error term in a regression model into an inefficiency component and a random error component.

The SFA incorporates the behavioral assumption that farmers will produce the maximal output from the available input factors. The approach therefore estimates a maximal milk output at the levels of a given set of input factors. The SFA method compares the potential and observed milk output for an individual farm. The highest potential milk output for the farm is estimated from the input factors and practices. This measure can be referred to as the maximal milk output on the frontier production curve.

The observed output represents how efficient the individual farmer is in using the appropriate techniques and the available set of input factors of cows, feed, labor, and veterinary and insemination services. The farmer's ability to detect reproductive disorders and his or her way of controlling them may affect efficiency. Therefore, observed milk output less than the potential maximal milk output will occur if the farmer is less efficient in using the best-practiced techniques and best set of input factors. This approach has been used in studies of production and cost functions in dairy farms (e.g., Kumbhakar et al., 1989; Bravo-Ureta and Rieger, 1990; Coelli, 1995; Hallam and Machado, 1996; Cuesta, 2000; Tauer, 2001; Lawson et al., 2003).

The inefficiency component as a function of possible explanatory factors can be investigated, as has been suggested by Kumbhakar et al. (1991) and Battese and Coelli (1995). We opted to include breed and housing variables in the inefficiency model. This ensured that the effects of these factors on efficiency were not ex- pressed by the reproductive disorder variables. It further allowed for testing if herds in these factor groups differed with regard to inefficiency. The age of the farmer was also included to represent other sources of inefficiency due to the different managerial abilities of the farmers.

\section{Econometric Model}

We have estimated 2 specific econometric models. Model 1 investigates reproductive disorders and model 2 investigates both reproductive disorders and reproductive management variables in the inefficiency functions. Models 1 and 2 are defined using the translog functional form for the milk production equation (Debertin, 1986).

The translog functional form is flexible and allows for milk output to display different production patterns. This functional form, unlike the Cobb-Douglas one, is not linear. The translog functional form, for example, displays the increasing scale production or increasing scale production at a decreasing rate. It further allows for variable substitution between input factors, whereas the Cobb-Douglas functional form restricts substitution between variables to one. The estimated elasticity coefficients express the weighted combination of the input factors that farmers used to produce milk. The estimates may differ at different stages of the estimated milk production function due to the second-order coefficients. For a normal milk production function, the first-order coefficient estimates lie between zero and one. The model for the translog functional form is formulated as:

$$
\begin{gathered}
\ln Y_{i}=\beta_{0}+\sum_{j=1}^{6} \beta_{j} \ln X_{j i}+1 / 2 \sum_{j=1}^{6} \sum_{k=1}^{6} \beta_{j k} \ln X_{j i} \ln X_{k i}+\varepsilon_{i} \\
(i=1, \ldots, 514) \\
\varepsilon_{i}=V_{i}-U_{i} \\
V_{i} \sim N\left(0, \sigma_{v}^{2}\right) \\
U_{i} \geq 0
\end{gathered}
$$

The $\ln$ indicates the natural logarithmic transformations; $Y_{i}$ is the energy-corrected milk output of farm $i$ of the 514 dairy farms, and $X_{j}$ indexes 1 to 6 input variables. The summary statistics and the description of symbols for variables in the milk production equation are provided in Table 1 . All of the $\beta$ parameters are to be estimated. The $\varepsilon_{i}$ is the error term and is composed of the random error $V_{i} \sim N\left(0, \sigma_{v}^{2}\right)$ and $U_{i}$, which represents the nonnegative random variables that account for inefficiency in milk production, such that, for the given technology and levels of inputs, the 
Table 1. Descriptive statistics for farm total production variables in the stochastic frontier production function and mean per cow per year $( \pm \mathrm{SE}){ }^{1}$

\begin{tabular}{llrrrrc}
\hline Variable & Symbol & \multicolumn{1}{c}{ Mean } & \multicolumn{1}{c}{ SE } & Minimum & Maximum & Per cow \\
\hline Energy-corrected milk (1000 kg) & $\mathrm{Y}$ & 512.26 & 11.29 & 33.82 & 1656.93 & $7158 \pm 41$ \\
Number of cows per herd & $\mathrm{x}_{1}$ & 71.00 & 2.00 & 5.00 & 216.00 & $\ldots$ \\
Concentrate (1000 feed units) & $\mathrm{x}_{2}$ & 152.81 & 4.06 & 1.79 & 672.04 & $2134 \pm 24$ \\
Roughage (1000 feed units) & $\mathrm{x}_{3}$ & 261.79 & 5.83 & 11.61 & 888.57 & $3725 \pm 37$ \\
Variable expenses (1000 DKK) & $\mathrm{x}_{4}$ & 67.42 & 1.72 & 6.21 & 234.40 & $952 \pm 15$ \\
Labor (man hours) & $\mathrm{x}_{5}$ & 2528 & 44 & 240 & 7373 & $38 \pm 0$ \\
Other Expenses (1000 DKK) & $\mathrm{x}_{6}$ & 168.30 & 4.04 & 11.14 & 809.77 & $2338 \pm 19$ \\
\hline
\end{tabular}

${ }^{1} \mathrm{Y}$ is milk delivered to the dairy processing plant in kilograms of energy-corrected milk; $\mathrm{x}_{1}$ is the number of cows per herd; $\mathrm{x}_{2}$ is the concentrate and cereals, where one feed unit $=1 \mathrm{~kg}$ of concentrate feed or cereals; $\mathrm{x}_{3}$ is total roughage feed units (e.g., one feed unit $=3.5 \mathrm{~kg}$ of maize silage); $\mathrm{x}_{4}$ is the sum of variable expenses, including the following inputs: 1 ) veterinary services and medication, 2) insemination services, 3) advisory control services, and 4) miscellaneous purchases that include straw for bedding but not feed. Variable expenses are measured in DKK (Danish kroner; $1 \mathrm{DKK}=$ about $\$ 0.13$ ). $\mathrm{x}_{5}$ is the available labor hours excluding labor for feed production and $\mathrm{x}_{6}$ is the sum of other expenses in DKK that represents depreciations, maintenance, and other miscellaneous non-allocative expenses (i.e., not recorded as variable expenses). This variable can be considered as a proxy for production equipment maintenance and quality.

observed output falls short of its potential output. The milk production inefficiency, $U_{i}$, is assumed to be distributed independently of $V_{i}$ and truncated from below at zero; for example $N^{+}\left(\mu_{i}, \sigma_{u}^{2}\right)$-distribution as in Battese and Coelli (1995), where $\mu_{i}$ is defined by:

$$
\begin{gathered}
\mu_{i}=\delta_{0}+\sum_{l=1}^{2} \alpha_{l} D_{l i}+\sum_{m=1}^{6} \delta_{m} Z_{m i}+\sum_{n=1}^{4} \omega_{n} W_{n i} \\
(i=1, \ldots, 514) .
\end{gathered}
$$

Where $D_{1}=1$ for Jersey herds, and $D_{1}=0$ for Holstein-Friesian, Red-Danish, and mixed-breed herds. We do not expect a major difference in the management of the heavier breeds compared with Jersey cows. A bivariate analysis was done to check this assumption. In this case $D_{2}=1$ for herds with loose housing, and $D_{2}=0$ for the tied housing. The $Z_{m}$ index 1 to 6 different reproductive disorders and $W_{n}$ index 1 is the age of the farmer and represents general management abilities (model 1 investigates only this part). The defined $W_{n}$ also indexes the 3 different reproductive management variables, of which $W_{2}$ is replacement rate. Of the 39,854 total cows calving in $1998,16 \%$ were culled in $1998,30 \%$ in $1999,17 \%$ in 2000 , and the rest, $37 \%$, were still in production beyond 2000 . Therefore, replacement rate is estimated as the number of cows at first calving in proportion to the total number of lactating cows, and more closely reflects the steadystate turnover of cows in a herd. The symbols $W_{3}$ and $W_{4}$ represent calving interval and cow age at first calving, respectively. Model 2 investigates the management variables numbered 2 to 4 in the inefficiency equation in addition to those investigated in model 1. All $\delta, \alpha$, and $\omega$ are the parameters to be estimated. The summary statistics and description of symbols for variables in the inefficiency equations are provided in Table 2.

The unknown variance parameters to be estimated are, in equation 1 , the variance associated with $\varepsilon_{i}$, the composed error defined as $\sigma_{\varepsilon}^{2}=\sigma_{v}^{2}+\sigma_{u}^{2}$, and in equation 2 the parameter associated with the variance of $U_{i}$ defined as $\gamma=\sigma_{u}^{2} / \sigma_{\varepsilon}^{2}$. The defined $\gamma$ has a value between 0 and 1, as described by Coelli et al. (1998). Basically, if inefficiency exists among farms, the estimated variance parameter $\gamma$ will be different from 0 . However, if the parameter is 0 , then the error term expresses the traditional random variation that is not under the control of the farmer.

The parameters of the model are estimated by the maximum-likelihood method using the FRONTIER 4.1 computer program (Coelli, 1996). The FRONTIER 4.1 program utilizes a 3 -step procedure. First, initial ordinary least squares estimates are obtained for the parameters in the production model. The second step involves a grid search for $\gamma$ with the $\beta$ parameters (except for $\left.\beta_{0}\right)$, while any other parameters $(\mu, \delta, \alpha$, and $\omega)$ are set to 0 . In this calculation $\beta_{0}$ and $\sigma_{\varepsilon}^{2}$ are adjusted (see Coelli et al., 1998). Lastly, values selected in the grid search are used in an iterative procedure to obtain the final maximum-likelihood estimates. Critical chi-squared values obtained from Kodde and Palm (1986) are used to test if the estimated $\gamma$ and the variables explaining inefficiency jointly equals 0 .

From the model specifications in equations 1 and 2, estimated MPE of the $i$ th herd is calculated as the observed milk output divided by the potential maximal milk output and measures their individual deviation from the frontier production curve. This is illustrated mathematically as: 
Table 2. Percentage distribution of farms by breed, housing system, induction of estrus and birth, mean, and quantile distribution incidence risks for farm reproductive disorders, and management variables in the inefficiency equation. ${ }^{1}$

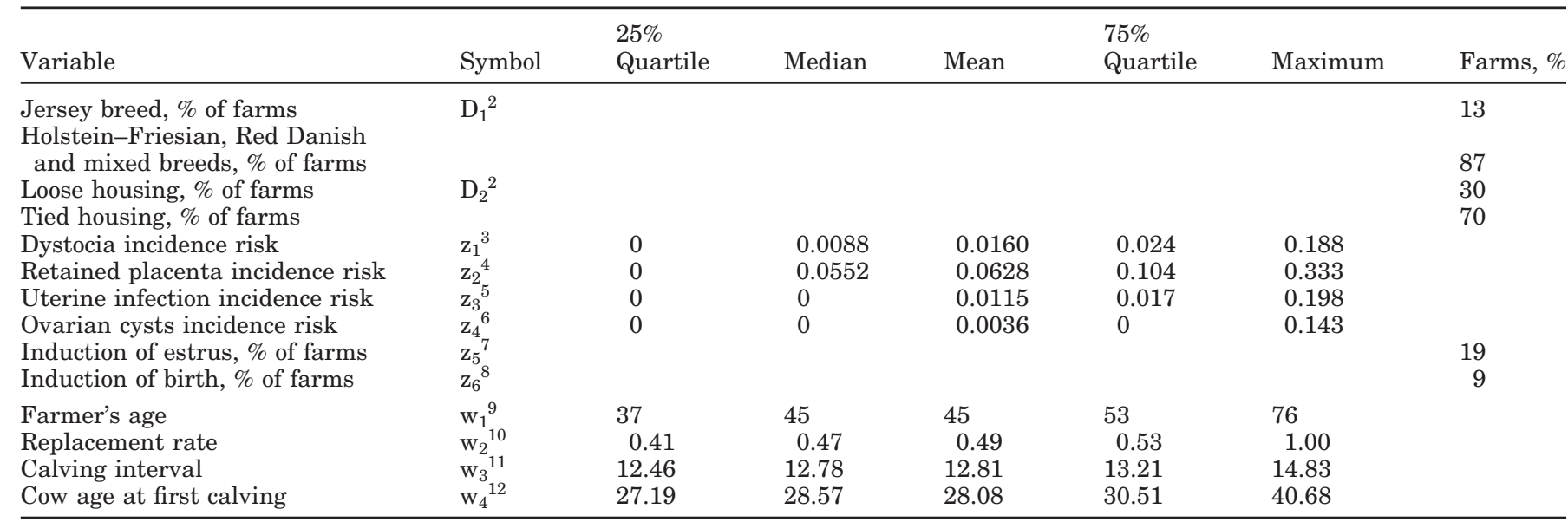

${ }^{1} \mathrm{D}_{1}=$ farm breed class, yes $=1$ for Jersey herds and no $=0$ for Friesian, Red-Danish, and mixed herds. We do not expect a major difference in the management of the heavier breeds compared with Jersey. A bivariate analysis was done to check this assumption.

${ }^{2} \mathrm{D}_{2}=$ farm housing system class, yes $=1$ for herds with loose housing systems and no $=0$ for the tied housing systems.

${ }^{3} \mathrm{z}_{1}=$ dystocia incidence risk. Dystocia is defined as reported cases of veterinary-assisted calving or performing caesarean in addition to uterine prolapse and uterine torsion.

${ }^{4} \mathrm{z}_{2}=$ incidence risk of retained placenta. This is the condition where the fetal membranes are not extruded from the uterus within 12 to $24 \mathrm{~h}$ after calving.

${ }^{5} z_{3}=$ incidence risk of uterine infections and includes metritis and vaginitis.

${ }^{6} \mathbf{z}_{4}=$ incidence risk of ovarian cyst, hormonal therapy and inactive ovaries.

${ }^{7} z_{5}=$ the percentage of farms with at least one case of induction of estrus (of silent heat and induction of estrus), yes $=1$ and no $=0$ in the analysis.

${ }^{8} z_{6}=$ the percentage of farms with at least one case of induction of birth, yes $=1$ and no $=0$ for analysis.

${ }^{9} \mathrm{w}_{1}=$ age of the farmer. This may reflect other management abilities.

${ }^{10} \mathrm{w}_{2}=$ number of cows at first calving in proportion of the total number of lactating cows in the year.

${ }^{11} \mathrm{w}_{3}=$ calving interval in months.

${ }^{12} \mathrm{w}_{4}=$ cow age at first calving in months.

$$
\begin{gathered}
M P E_{i}=\frac{Y_{i}}{Y_{i}^{*}} \\
=\frac{\exp \left(\beta_{0}+\sum_{j=1}^{6} \beta_{j} \ln X_{j i}+1 / 2 \sum_{j=1}^{6} \sum_{k=1}^{6} \beta_{j k} \ln X_{j i} \ln X_{k i}+V_{i}-U_{i}\right)}{\exp \left(\beta_{0}+\sum_{j=1}^{6} \beta_{j} \ln X_{j i}+1 / 2 \sum_{j=1}^{6} \sum_{k=1}^{6} \beta_{j k} \ln X_{j i} \ln X_{k i}+V_{i}\right)} \\
=\exp \left(-U_{i}\right),
\end{gathered}
$$

where $Y_{i}^{*}$ is the estimated maximal energy-corrected milk output and the other variables are defined as mentioned earlier. The milk production inefficiency part of the total error in this study is estimated as proposed in Battese and Coelli (1988).

The 2 milk output measures are modeled and estimated in a set-up including a production function and an inefficiency function. The production function contains the relationships between milk output and the set of input factors (Table 1), whereas the inefficiency function includes reproductive disorders and reproductive management variables in Table 2 that might explain the variation in the farms' inefficiencies. This procedure, which allows frontier and efficiency functions to vary by input variables and reproductive disorders, is referred to as stochastic frontier production function analysis. For the milk production function in equation 1 , the elasticities are calculated by differentiating the function. For example, the elasticity of milk output with respect to the number of cows $\left(X_{1}\right)$ is given by:

$$
\begin{gathered}
\partial \ln Y / \partial 1 \mathrm{n} X_{1}=\beta_{1}+\beta_{11} \ln X_{1}+\beta_{12} \ln X_{2} \\
+\beta_{13} \ln X_{3}+\beta_{14} 1 \mathrm{n} X_{4}+\beta_{15} \ln X_{5}+\beta_{16} \ln X_{6} .
\end{gathered}
$$

The estimate obtained from equation 4 using mean values of $\ln \mathrm{X}_{1}$ to $\ln \mathrm{X}_{6}$, for the variable $\mathrm{X}_{1}$ to $\mathrm{X}_{6}$ in Table 1 is referred to in the study as first-order coefficients ( $\beta_{1}$ to $\beta_{6}$ in Table 3 ). This estimate is quickly obtained when the values in equation 1 are mean-corrected before estimation. Cuesta (2000) used a similar method and this was checked and found to be correct for the data.

Testing for the statistical significance of a single or multiple number of parameters in the models was 
Table 3. Translog SFA maximum-likelihood estimates in order, of elasticities $\left(\beta_{1}-\beta_{6}\right)$, quadratic $\left(\beta_{11}-\beta_{66}\right)$ and interactions $\left(\beta_{12}-\beta_{56}\right)$ terms for the stochastic frontier production function.

\begin{tabular}{|c|c|c|c|c|c|}
\hline \multirow[b]{2}{*}{ Variables } & \multirow[b]{2}{*}{ Parameters } & \multicolumn{2}{|c|}{ Model 1} & \multicolumn{2}{|c|}{ Model $2^{1}$} \\
\hline & & Coefficients & $\mathrm{SE}^{2}$ & Coefficients & $\mathrm{SE}$ \\
\hline Constant & $\beta_{0}$ & $0.085^{* *}$ & 0.009 & $0.083^{* *}$ & 0.010 \\
\hline Number of cows & $\beta_{1}$ & $0.523^{* *}$ & 0.046 & $0.555^{* *}$ & 0.045 \\
\hline Concentrate & $\beta_{2}$ & $0.159 * *$ & 0.018 & $0.156 * *$ & 0.018 \\
\hline Roughage & $\beta_{3}$ & $0.082^{* *}$ & 0.022 & $0.076^{* *}$ & 0.022 \\
\hline Variable expenses & $\beta_{4}$ & $0.088^{* *}$ & 0.014 & $0.086 * *$ & 0.015 \\
\hline Labor & $\beta_{5}$ & $0.085^{* *}$ & 0.031 & $0.082^{* *}$ & 0.029 \\
\hline Other expenses & $\beta_{6}$ & $0.097 * *$ & 0.026 & $0.081 * *$ & 0.025 \\
\hline$(\text { Number of cows })^{2}$ & $\beta_{11}$ & $-1.623 * *$ & 0.618 & $-1.420 *$ & 0.595 \\
\hline$(\text { Concentrate })^{2}$ & $\beta_{22}$ & $0.038 \dagger$ & 0.023 & $0.040 \dagger$ & 0.022 \\
\hline (Roughage) $^{2}$ & $\beta_{33}$ & -0.067 & 0.127 & -0.063 & 0.122 \\
\hline (Variable expenses) $^{2}$ & $\beta_{44}$ & -0.049 & 0.045 & -0.032 & 0.046 \\
\hline$(\text { Labor })^{2}$ & $\beta_{55}$ & -0.117 & 0.204 & -0.164 & 0.199 \\
\hline$(\text { Other expenses })^{2}$ & $\beta_{66}$ & -0.041 & 0.203 & -0.043 & 0.198 \\
\hline (Number of cows $) \times($ concentrate $)$ & $\beta_{12}$ & $0.466^{* *}$ & 0.146 & $0.399 * *$ & 0.145 \\
\hline (Number of cows $) \times($ roughage $)$ & $\beta_{13}$ & $0.456 \dagger$ & 0.236 & $0.411 \dagger$ & 0.229 \\
\hline (Number of cows $) \times($ variable expenses $)$ & $\beta_{14}$ & -0.021 & 0.112 & -0.055 & 0.111 \\
\hline (Number of cows $) \times($ labor $)$ & $\beta_{15}$ & -0.115 & 0.277 & -0.183 & 0.265 \\
\hline (Number of cows $) \times($ other expenses $)$ & $\beta_{16}$ & $0.587^{*}$ & 0.283 & $0.573^{*}$ & 0.275 \\
\hline$($ Concentrate $) \times($ roughage $)$ & $\beta_{23}$ & $-0.146 \dagger$ & 0.081 & -0.110 & 0.080 \\
\hline$($ Concentrate $) \times($ variable expenses $)$ & $\beta_{24}$ & $-0.098^{*}$ & 0.044 & $-0.093^{*}$ & 0.045 \\
\hline (Concentrate) $\times$ (labor) & $\beta_{25}$ & 0.111 & 0.117 & 0.138 & 0.113 \\
\hline$($ Concentrate $) \times($ other expenses $)$ & $\beta_{26}$ & $-0.337 * *$ & 0.086 & $-0.337 * *$ & 0.088 \\
\hline (Roughage) $\times($ variable expenses $)$ & $\beta_{34}$ & -0.032 & 0.056 & -0.027 & 0.056 \\
\hline (Roughage $) \times($ labor $)$ & $\beta_{35}$ & 0.047 & 0.130 & 0.083 & 0.121 \\
\hline (Roughage) × (other expenses) & $\beta_{36}$ & -0.117 & 0.126 & -0.145 & 0.121 \\
\hline (Variable cost $) \times($ labor $)$ & $\beta_{45}$ & 0.080 & 0.091 & 0.057 & 0.090 \\
\hline (Variable expenses $) \times($ other expenses $)$ & $\beta_{46}$ & 0.090 & 0.075 & 0.104 & 0.075 \\
\hline$($ Labor $) \times($ other expenses $)$ & $\beta_{56}$ & -0.064 & 0.179 & 0.003 & 0.173 \\
\hline $\begin{array}{l}* *(P<0.01) \\
*(P<0.05) \\
\dagger(P<0.10) \\
1 \text { The model }\end{array}$ & & & & & \\
\hline Note: results for both models are cont & a Table 4 & & & & \\
\hline
\end{tabular}

conducted using the generalized likelihood ratio statistic, defined as:

$$
\lambda=-2 \ln \left(L\left(H_{0}\right) / L\left(H_{1}\right)\right),
$$

where $L\left(H_{0}\right)$ is the likelihood function for the null hypothesis and $L\left(H_{1}\right)$ is the likelihood function for the unrestricted model. The unrestricted model is the one specified in equations 1 and 2 , which is to be estimated simultaneously. The first null hypothesis $H_{0}$ is that equation 2 equals 0 . A second $H_{0}$ is that the quadratic and the interaction terms jointly equal zero when tested against the unrestricted model. If the null hypothesis is true, then $\lambda$ has approximately a chisquared (or mixed chi-squared) distribution with the degrees of freedom equal to the difference between the number of parameters estimated under $H_{1}$ and $H_{0}$ (Coelli et al., 1998).

\section{RESULTS}

The Stochastic Frontier Production Equation

The maximum-likelihood estimates for the parameters in the translog stochastic frontier production function for Danish dairy farmers are presented in Tables 3 and 4 . In order to complement the interpretations and the understanding of the estimates in Table 4, supplementary analyses were done. The first is a regression analysis (i.e., of reproductive management variables on each reproductive disorder). The second is Pearson correlation analysis used to investigate the relationship between all the inefficiency explanatory variables. Thirdly, the means of reproductive disorders and management variables for levels of MPE score groups, 60 to 90,90 to 95 , and 95 to 99 for low, medium, and high scores, were compared. Groups based on MPE scores were created after multiplying the efficiency estimates by 100 . 
Table 4. Maximum-likelihood estimates for breed, housing, and incidence risk and management variables in the inefficiency function equation and the distribution of MPE scores.

\begin{tabular}{|c|c|c|c|c|c|}
\hline \multirow[b]{2}{*}{ Variables } & \multirow[b]{2}{*}{ Parameters } & \multicolumn{2}{|c|}{ Model 1} & \multicolumn{2}{|c|}{ Model $2^{1}$} \\
\hline & & Coefficients $^{2}$ & $\mathrm{SE}^{3}$ & Coefficients $^{2}$ & $\mathrm{SE}$ \\
\hline Constant & $\delta_{0}$ & $-1.28 \dagger$ & 0.763 & -2.376 & 1.204 \\
\hline Jersey breed & $\alpha_{1}$ & -0.147 & 0.105 & 0.140 & 0.088 \\
\hline Loose housing-system & $\alpha_{2}$ & 0.045 & 0.036 & $0.081 \dagger$ & 0.047 \\
\hline Dystocia incidence risk & $\delta_{1}$ & 0.674 & 0.900 & 1.398 & 1.134 \\
\hline Retained placenta incidence risk & $\delta_{2}$ & $-2.221 \dagger$ & 1.157 & -0.420 & 0.331 \\
\hline Uterine infection incidence risk & $\delta_{3}$ & -4.144 & 2.924 & 0.190 & 1.608 \\
\hline Ovarian cyst incidence risk & $\delta_{4}$ & -8.937 & 5.959 & -3.810 & 6.438 \\
\hline Induction-of-estrus dummy & $\delta_{5}$ & $-0.192 \dagger$ & 0.112 & -0.063 & 0.043 \\
\hline Induction-of-birth dummy & $\delta_{6}$ & -0.582 & 0.380 & -0.261 & 0.250 \\
\hline Farmers' age & $\omega_{1}$ & $0.015^{*}$ & 0.007 & $0.0071 \dagger$ & 0.0040 \\
\hline Replacement rate & $\omega_{2}$ & $\ldots$ & $\ldots$ & -0.0692 & 0.1085 \\
\hline Calving interval & $\omega_{3}$ & $\ldots$ & $\ldots$ & 0.0108 & 0.0247 \\
\hline Cow age at first calving & $\omega_{4}$ & & & $0.0555^{*}$ & 0.0237 \\
\hline Error variance & $\sigma_{\varepsilon}$ & $0.082^{*}$ & 0.038 & $0.0368^{*}$ & 0.0156 \\
\hline Variance parameter ${ }^{4}$ & $\gamma$ & $0.940^{* *}$ & 0.023 & $0.8643^{* *}$ & 0.0597 \\
\hline Log-likelihood value ${ }^{5}$ & & 460.78 & $\ldots$ & 480.30 & $\ldots$ \\
\hline Log-likelihood value ${ }^{6}$ & & 436.16 & $\ldots$ & 436.16 & $\ldots$ \\
\hline Log-likelihood value $^{7}$ & & 428.40 & $\ldots$ & 447.84 & $\ldots$ \\
\hline \multicolumn{6}{|l|}{ Distribution of technical efficiency } \\
\hline Mean MPE $(\times 100)$ & & 92.7 & $\ldots$ & 92.9 & $\ldots$ \\
\hline Min. MPE $(\times 100)$ & & 60.0 & & 62.2 & \\
\hline Max. MPE $(\times 100)$ & & 98.4 & & 98.4 & \\
\hline \multicolumn{6}{|l|}{$* *(P<0.01)$} \\
\hline \multicolumn{6}{|l|}{$*(P<0.05)$} \\
\hline$\dagger(P<0.10)$ & & & & & \\
\hline \multicolumn{6}{|l|}{${ }^{1}$ Model with reproductive disorde } \\
\hline \multicolumn{6}{|c|}{ Note: results for both models are continued from Table 3 . } \\
\hline \multicolumn{6}{|c|}{${ }^{2}$ Negative signs of coefficients represent reduction in inefficiency (or increase in efficiency). } \\
\hline \multicolumn{6}{|c|}{${ }^{3}$ Standard error of mean. } \\
\hline \multicolumn{6}{|c|}{$\begin{array}{l}{ }^{4} \text { Gamma is the variance parameter associated with inefficiency. It is zero if no inefficiency exists in the } \\
\text { traditional error term. }\end{array}$} \\
\hline \multicolumn{6}{|c|}{${ }^{5}$ For the unrestricted model for production and inefficiency equations. } \\
\hline \multicolumn{6}{|c|}{${ }^{6}$ For the restricted model without the inefficiency equation. } \\
\hline $\mathrm{r}$ the restricted model for & & & & & \\
\hline
\end{tabular}

In Table 3 , all of the first-order coefficients $\left(\beta_{1}\right.$ to $\left.\beta_{6}\right)$ for the input factors are positive and different from zero $(P<0.01)$. These values are the elasticities evaluated at the sample means because the output and input variables were mean-corrected before estimation. For model 1 , the estimated coefficient, 0.523 for $\beta_{1}$, is the marginal impact for the number of cows and can be interpreted as a $0.5 \%$ (at one decimal place) increase in milk output due to a $1 \%$ increase in the number of cows. The $0.5 \%$ is estimated, whereas the other inputs are held constant. For the mean values shown in Table 1 , this implies that one additional cow, which is approximately $1.41 \%$ of 71 cows, would result in a marginal 3778-kg increase in mean energy-corrected milk output $([512,260 \mathrm{~kg}$ of milk $\times 0.01] \times 0.523 \times 1.41)$. It should be emphasized that this calculated estimate is not related to the average milk output per cow but to the marginal increase of the farm milk output. The approximated financial implication of these values is realized by comparing the income from the extra milk output and the costs of the input. For example, the accrued income for the marginal increase in milk output mentioned above is 9067 Danish kroners (DKK) $(3778 \times 2.40$ DKK [farm average milk price in 1998; $1 \mathrm{DKK}=$ about $\$ 0.13]$ ). It suggests that the price paid and the cost incurred to acquire the cow should not exceed 9067 DKK (approximately $\$ 1180$ ). The average cost of a cow was 5234 DKK (approximately \$680) in 1998, which suggests that the extra investment will be profitable if the capacity to have an extra cow exists on the farm. Similarly, corresponding marginal values could be calculated for the other input variables with mean values.

The sum of the six first-order milk output elasticities $\left(\beta_{1}\right.$ to $\beta_{6}$ ) in Table 3 for model 1 is 1.034 . It suggests that increasing all inputs by $1 \%$ will increase average 
farm output productivity by $3 \%$ (i.e., the increase in the ratio of total milk output divided by the sum of all inputs). This is referred to as the scale elasticity, and it is at the mean values of the input set. However, because the pattern of the translog production function is increasing at a decreasing rate, the scale elasticity like the individual first-order coefficients differs at various levels of the input sets. For example, the estimated scale elasticity is 1.00 for the farm with a MPE score of 80 , and for the farm with a MPE score of 98 , it is 1.09 (at 2 decimal places). The relative percentage of weight for input estimates making up the scale elasticity is further covered in the discussion as an example for the 2 farms.

For model 1 second-order coefficients in Table 3, three coefficients $\left(\beta_{11}, \beta_{12}\right.$, and $\left.\beta_{26}\right)$ are different from zero $(P<0.01), 2$ other coefficients $\left(\beta_{16}\right.$ and $\left.\beta_{24}\right)$ are different from zero $(P<0.05)$ and an additional three coefficients $\left(\beta_{22}, \beta_{13}\right.$, and $\left.\beta_{23}\right)$ are different from zero $(P<0.10)$. The values and the signs associated $\beta_{11}$ and $\beta_{12}$ to $\beta_{16}$ reflect in part why $\beta_{1}$ is $0.5 \%$ (and not $1 \%$ ), which is the outcome of equation 4 . That $\beta_{23}$ is negative suggests that farmers substitute concentrate and roughage feed, which should be expected as prices vary for those inputs.

Similar levels of significance for input variables are seen for model 2 (Table 3) (i.e., after the inclusion of reproductive management variables in the inefficiency model). However, a closer look at the estimates shows that the coefficient estimate $\beta_{1}$, for the number of cows, is 3.2 percentage units higher for model 2 vs. model 1. This difference is compensated for by lower estimates for the coefficients $\left(\beta_{2}\right.$ to $\left.\beta_{6}\right)$ in model 2 . In practical terms, it indicates that the marginal increase in milk output for an additional cow is underestimated by $231 \mathrm{~kg}$ of energy-corrected milk when reproductive management variables are not included in the model. This is the difference between the $3778 \mathrm{~kg}$ mentioned above and the $4009 \mathrm{~kg}$ for model 2 (i.e., the impact for $\beta_{1}$ ). A closer look at $\omega_{1}$ to $\omega_{4}$ in Table 4 suggests that replacement rate, which has the only negative parameter coefficient (i.e., reducing inefficiency) contributed to the increase in the number of cows' estimate in model 2. However, the estimated coefficient is not significant.

For the model specifications 1 and 2, the joint null hypothesis $H_{0}$ is that no inefficiency exists in Danish dairy farms (i.e., the parameter $\gamma$ and all the parameters in equation 2 , that is $\delta_{0}$ to $\omega_{5}$ are jointly equal to zero). This was rejected $(P<0.01)$ and suggests that one or more of the parameters tested are different from zero. From equation 5, the FRONTIER program provides the likelihood ratios for this test. The rejection indicates that the ordinary least squares regres- sion model is not the best fit for the data given the translog stochastic frontier formulation. The significance of the $\gamma$ estimates (Table 4) alone indicates that the traditional error term includes an inefficiency random component. It suggests that individual efficiency scores can be calculated to identify the high- and lowefficiency farms, estimate the magnitude of inefficiency in terms of milk output, and investigate the sources of inefficiency in Danish dairy herds. This is the objective of equation 2 in models 1 and 2 .

A null hypothesis that the second-order coefficients $\left(\beta_{11}\right.$ to $\left.\beta_{56}\right)$ are jointly zero is a test of a linearly increasing production function pattern (i.e., the simpler CobbDouglas functional form, which is a special case of the translog form). The tests for both models are rejected $(P<0.01)$ and indicate that the translog functional is a better fit for data.

\section{The Inefficiency Equation}

The estimated MPE and the $\delta, \alpha$, and $\omega$ estimates for the inefficiency model in Table 4 are of special interest. Note: A positive estimate indicates a lower MPE. The mean MPE score is 93, with a range of 60 to 98 (with zero decimal places). Milk production efficiency ranges from 0 to 1 and can be expressed as percentages or decimals. Therefore, the average milk production inefficiency is 0.07 (1-MPE), which reflects a marginal average loss of $550 \mathrm{~kg}$ of energy-corrected milk per cow. This value was calculated as the average of the estimated individual farm milk losses per cow and is therefore slightly higher than $501 \mathrm{~kg}$, which was obtained from Table 1 as $7158 \mathrm{~kg}$ of milk output per cow multiplied by an inefficiency value of 0.07 .

For model 1, about $19 \%$ of the farms had an efficiency score below $90 \%$, and this is similar for model 2 , which included the reproductive management factors. The $\alpha_{1}$ coefficient is not different from zero, but $\alpha_{2}$ is $(P<$ 0.10 ), which suggests that there are no differences in inefficiency scores for farms with Jersey and other breeds. However, farms with loose housing systems tend to be less efficient compared with farms with tied housing systems.

Before inclusion of reproductive management factors, the coefficient for dystocia incidence was not significantly different from zero. The estimated coefficients for the incidence risk of retained placenta $\left(\delta_{2}\right)$ and induction of estrus $\left(\delta_{5}\right)$ are negative and different from zero $(P<0.10)$. It suggests that farms reporting high levels of retained placenta and farms practicing induction of estrus are also more efficient in production. The coefficients for uterine infection $\left(\delta_{3}\right)$, ovarian cysts $\left(\delta_{4}\right)$, incidence risks, and birth induction $\left(\delta_{6}\right)$, are not different from zero. It suggests that these disorders 
Table 5. Number of farms, mean and SE for low, medium, and high milk production efficiency (MPE) Scores ${ }^{1}$.

\begin{tabular}{|c|c|c|c|c|c|c|}
\hline MPE scores grouping: & Low $(60$ & to 90$)$ & Medium (9 & to 95$)$ & High ( & 95) \\
\hline \multirow[t]{2}{*}{ Number of farms } & \multicolumn{2}{|c|}{94} & \multicolumn{2}{|c|}{181} & \multicolumn{2}{|c|}{239} \\
\hline & Mean & $\mathrm{SE}^{2}$ & Mean & SE & Mean & $\mathrm{SE}$ \\
\hline Mean MPE $(\times 100)$ & 83.3 & 0.69 & 93.1 & 0.10 & 96.5 & 0.05 \\
\hline Energy-corrected milk per cow & $5931^{\mathrm{c}}$ & 87 & $6908^{b}$ & 35 & $7831^{\mathrm{a}}$ & 38 \\
\hline Number of cows per farm & $64^{\mathrm{b}}$ & 4 & $74^{\mathrm{a}}$ & 3 & $72^{\mathrm{a}}$ & 2 \\
\hline \multicolumn{7}{|l|}{$\begin{array}{l}\text { Production function variables } \\
\text { Input per cow }\end{array}$} \\
\hline Concentrate feed unit & $2091^{\mathrm{a}}$ & 61 & $2123^{\mathrm{a}}$ & 43 & $2159^{\mathrm{a}}$ & 34 \\
\hline Roughage feed unit & $3699^{\mathrm{a}}$ & 89 & $3694^{\mathrm{a}}$ & 68 & $3764^{\mathrm{a}}$ & 48 \\
\hline Variable expenses in $\mathrm{DKK}^{3}$ & $857^{\mathrm{b}}$ & 43 & $903^{\mathrm{b}}$ & 21 & $1027^{\mathrm{a}}$ & 21 \\
\hline Labor hours & $40^{\mathrm{a}}$ & 1 & $37^{\mathrm{b}}$ & 1 & $37^{\mathrm{b}}$ & 1 \\
\hline Other expenses in DKK & $2286^{b}$ & 53 & $2284^{\mathrm{b}}$ & 31 & $2400^{\mathrm{a}}$ & 27 \\
\hline \multicolumn{7}{|l|}{ Inefficiency function variables } \\
\hline Dystocia incidence risk & $0.0136^{\mathrm{a}}$ & 0.0026 & $0.0172^{\mathrm{a}}$ & 0.0018 & $0.0161^{\mathrm{a}}$ & 0.0013 \\
\hline Retained placenta incidence risk & $0.0469^{\mathrm{b}}$ & 0.0063 & $0.0580^{\mathrm{b}}$ & 0.0043 & $0.0727^{\mathrm{a}}$ & 0.0037 \\
\hline Uterine infection incidence risk & $0.0100^{\mathrm{b}}$ & 0.0022 & $0.0092^{\mathrm{ab}}$ & 0.0013 & $0.0138^{\mathrm{a}}$ & 0.0015 \\
\hline Ovarian cysts incidence risk & $0.0010^{\mathrm{b}}$ & 0 & $0.0018^{\mathrm{b}}$ & 0 & $0.0060^{\mathrm{a}}$ & 0.0011 \\
\hline Induction of estrus, $\%$ of farms & $10^{\mathrm{b}}$ & & $12^{\mathrm{b}}$ & & $28^{\mathrm{a}}$ & \\
\hline Induction of birth, $\%$ of farms & $1^{\mathrm{c}}$ & & $7^{\mathrm{b}}$ & & $13^{\mathrm{a}}$ & \\
\hline Farmer's age & $49^{\mathrm{a}}$ & 1 & $47^{\mathrm{a}}$ & 1 & $44^{\mathrm{b}}$ & 1 \\
\hline Replacement rate ${ }^{4}$ & $0.5265^{\mathrm{b}}$ & 0.0240 & $0.4907^{\mathrm{a}}$ & 0.0100 & $0.4702^{\mathrm{a}}$ & 0.0068 \\
\hline Calving interval & $12.79^{\mathrm{ab}}$ & 0.080 & $12.73^{\mathrm{a}}$ & 0.052 & $12.88^{\mathrm{b}}$ & 0.036 \\
\hline Cow age at first calving & $31.05^{\mathrm{a}}$ & 0.374 & $29.19^{\mathrm{b}}$ & 0.198 & $28.22^{\mathrm{c}}$ & 0.139 \\
\hline
\end{tabular}

abc Means with differing letter within a row differ significantly $(P<0.05)$.

${ }^{1}$ Efficiency scores from stochastic frontier analysis are used to group the farms into low, medium, and high efficiency herds.

2 The standard error of mean.

${ }^{3}$ DKK $=$ Danish kroner; 1 DKK = about $\$ 0.13$.

${ }^{4}$ The number of cows at first calving in proportion of the total number of lactating cows in the year.

are not correlated with MPE. The coefficient farmers' age $\left(\omega_{1}\right)$ is significant and suggests that older farmers are less efficient.

For model 2, after inclusion of reproductive management variables, none of the disease variables is significantly correlated with inefficiency. The joint test that the reproductive disorders $\left(\delta_{1}\right.$ to $\left.\delta_{6}\right)$ equal zero could not be rejected $(P>0.33)$. It suggests that reproductive disorder variables do not explain the variation in inefficiency. The positive coefficient for the farmer's age $\left(\omega_{1}\right)$ is different from zero $(P<0.10)$ and quite close to the one estimated in model 1.

The reproductive management factor, replacement rate $\left(\omega_{2}\right)$, is not different from zero. However, the estimated coefficient for cow age at first calving $\left(\omega_{4}\right)$ is different from zero $(P<0.05)$. The positive estimate indicates that, on average, farms with higher cow ages at first calving are farms with lower MPE scores. This could reflect higher overall management ability, with better heifer development programs being associated with subsequent measures of production efficiency. High-efficiency herds that manage to have first calvings at younger ages also may maintain maximum milk output in part because they have the possibility to replace cows with reproductive disorders. This implies that farmers should ensure the availability of bred heifers developed and bred to calve at relatively young ages to replace cows with reproductive disorders.

The positive association between the farmer's age and inefficiency in model 1 suggests that young farmers are better at utilizing factor inputs to maximize milk output. Table 5 shows that younger farmers manage the herds with the highest productivity per cow. These farms use the same level of concentrate and roughage per cow as the less efficient groups (Table $5)$. The higher efficiency could be the result of the adoption of improved methods of production and new investments in production systems, which is reflected by the high estimate for other expenses.

\section{DISCUSSION}

\section{Efficiency}

The efficiency scores obtained from SFA and expressed in percentages suggests that milk loss among Danish dairy farmers is as high as $40 \%$ and as low as 1\%. From the MPE scores, it is calculated that milk 
loss on average is 1142,488 , and $256 \mathrm{~kg}$ of energycorrected milk per cow, respectively, for the low-, medium-, and high-MPE score groups. It is easy to suggest the presence of inefficiency and to calculate the associated loss, but what is more important is to identify sources of inefficiency. Reproductive disorders and management variables have been related to the estimated MPE in an attempt to identify the sources of inefficiency. In addition, the coefficients $\beta_{1}$ to $\beta_{6}$ provide information to evaluate how the available sets of inputs have been combined to attain maximal milk output. The value of a 0.5 -percentage-unit increase in milk output due to a 1-percentage-unit increase in the number of cows is estimated, whereas all other inputs are held constant. It is the marginal output value, which is always less than the corresponding average output. It should be any value above zero, but it is usually expected to lie between 0 and $1 \%$ at the stage of maximal milk production. It may be $1 \%$ when the other factors are zero.

The 0.5 percentage-unit increase also expresses the weight of the number of cows in the scale elasticity of 1.034 (i.e., the sum of the elasticity coefficients $\beta_{1}$ to $\left.\beta_{6}\right)$. For example, the weight is $51 \%$ ([0.523/1.034] $\times$ 100) for the number of cows, whereas concentrate feed $(15 \%)$, roughage $(8 \%)$, variable expenses $(9 \%)$, labor $(7 \%)$, and other expenses (10\%) make up the balance. Dairy management advisors can use these weightings and in addition to the results from the inefficiency analysis, compare and advise low- and high-MPE farmers on how to improve or maintain milk output. The weights of inputs calculated for a farm with a MPE score of 80 and 98 , representing the 5th and the 99th percentiles of MPE scores, are illustrated in Figure 1.

As shown in Figure 1, the farm with a MPE score of 80 (left bar of pair) has a combination of inputs that is weighted toward concentrate and roughage feed consumption in addition to variable expenses. These weights are high compared with the farm with a MPE score of 98 . However, the weight attributed to the output elasticity ( 0.5 percentage units) for the number of cows is low compared with that of the farm with a MPE score of 98, which had a of cow output elasticity of $0.7 \%$. (Note that the 0.5 and $0.7 \%$ elasticities are estimated using the output and input values of the 2 farms.) For a farm advisor, it may suggest that the farm with MPE score of 80 seems to be producing less milk from its cows, even though feed consumption is high. This evaluation may form the basis for dialog between the farmer with a MPE score of 80 and the advisor in an attempt to improve MPE (all else being equal).

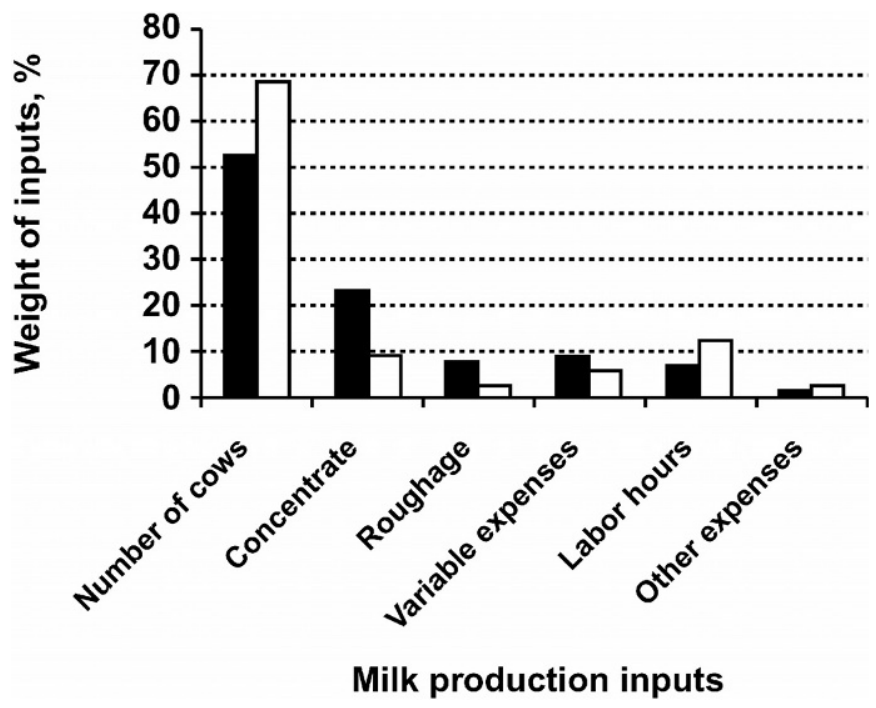

Figure 1. Weight of inputs in scale elasticity of 1.00 for the herd with milk production efficiency (MPE) score 80 (left bar of pair), and in the scale elasticity of 1.09 for the herd with MPE score 98 (right bar of pair).

Efficiency analysis can also be used in farm comparative studies in the use of new production techniques and to evaluate farmers' economic problems arising from political decisions, such as the removal of economic subsidies, milk quota regulations, and environmental regulations.

\section{Production Equation}

The positive direction of estimates $\left(\beta_{1}\right.$ to $\left.\beta_{6}\right)$ is as expected from the stochastic frontier production model and are quite close to those obtained by Lawson et al. (2003). However, we note that the marginal elasticity of concentrate feed is double that for roughage feed, which tends to be inconsistent with the estimates obtained in Lawson et al. (2003), where the values are the same. This probably reflects the degree to which the substitution between concentrate feed and roughage was used by farmers in 1998 .

The highly skewed distribution of the MPE scores shown in Figure 2, with most farms at the more efficient end, tends to indicate a fairly homogenous production technology in the Danish dairy industry. In addition, $19 \%$ of the farms have efficiency scores below $90 \%$, and this is similar to the Danish study by Lawson et al. (2003). The mean MPE in this study is higher than the technical efficiencies (MPE) reported by Cuesta (2000), Hallam and Machado (1996), and Bravo-Ureta and Rieger (1991), for Spanish, Portuguese, and US data, respectively. It is difficult to explain these differences, as efficiency scores are depen- 


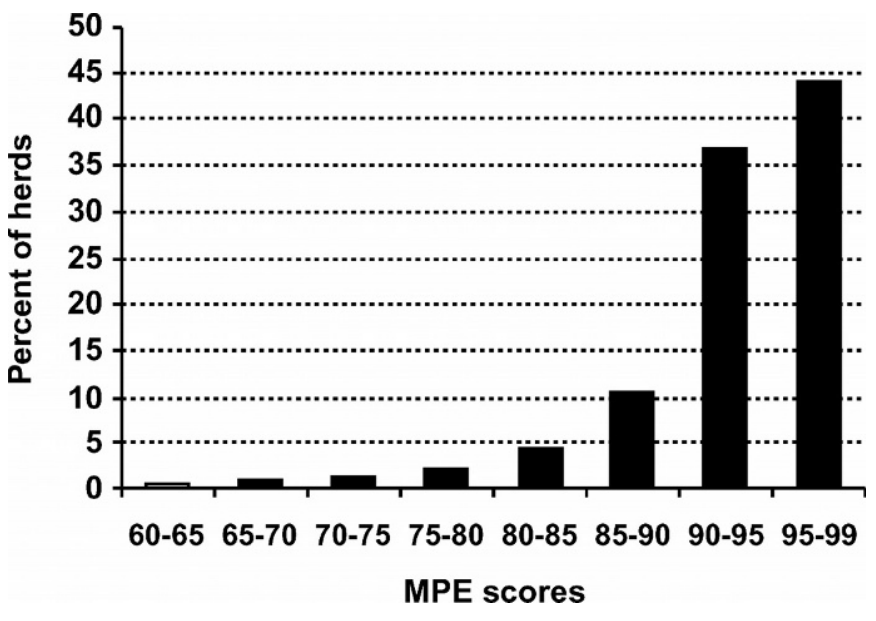

Figure 2. Distribution of herds at various milk production efficiency (MPE) scores.

dent on the data used in analysis and other factors (e.g., the similarity of Danish dairy farms). In areas where farms are more variable, most farms seem to be less efficient than the most efficient farms. Therefore, in those places, the mean MPE is lower than the mean MPE on Danish dairy farms.

\section{Inefficiency Equation}

The dystocia incidence risk (1.6\%) in this study is close to the $2.2 \%$ reported by Bruun et al. (2002) and is within the range of 1.3 to $14 \%$ given by Fourichon (1999). The average estimated incidence risk for uterine infection (metritis and vagnitis) is $1.2 \%$ and is close to the $0.7 \%$ estimate and range 1 to $21 \%$ given by Bruun et al. (2002) for metritis only. However, our estimate is low relative to the 3 to $45 \%$ range given by Bartlet et al. (1986), Fourichon et al. (1999), and Østergaard and Gröhn (1999). The incidence risk for ovarian cysts $(0.4 \%)$ is relatively low compared with the ranges of 3.7 to $16 \%$ given in Fourichon et al. (1999) and the 9.4 to $10.5 \%$ reported by Østergaard and Gröhn (1999). However, because incidences of cystic ovaries were from within 1998 for cows calving in the same year, the actual percentage of cystic ovaries across complete lactations could be higher.

The incidence risk of retained placenta $(6.3 \%)$ in this study is within the range of 2 to $18 \%$ given by Bartlett et al. (1986), the range of 3.1 to $18.0 \%$ given by Fourichon et al. (1999), and similar to the estimate of $6.6 \%$ by Bruun et al. (2002), but was lower compared with the range of 9 to $12 \%$ reported for 3 research herds by Østergaard and Gröhn (1999). Because retained placenta is correlated with most cases of other reproductive disorders and accounts for the greater propor- tion of treated and reported cases, it can generally best explain the relationship between reproductive disorders (except for dystocia) and milk production efficiency. Therefore, the other estimates should be evaluated with care.

We initially expected inefficient herds to report more cases of reproductive disorders, but the result turned out to be the opposite. The efficient herds also enroll relatively younger cows in production and tend to use the practice of induction of estrus. This management routine may be compensation for negative effect of reproductive diseases and disorders. This is particularly true for cases of persistent ovarian luteal cysts, which can be removed by prostaglandin, often used for induction of estrus. Similarly, treatment for induction of estrus cleans the reproductive system and may prevent infectious disorders such as metritis. Fourichon et al. (2000) found that dystocia, retained placenta, metritis, cystic ovaries, anestrus, and abortion were associated with an increased number of days to first service, a lower conception rate, and thus, more days to conception. The review study by Fourichon et al. (1999) revealed that for a full lactation period or for only 100 to $119 \mathrm{~d}$ of the lactation period, milk loss due to reproductive disorders ranges from 0.3 to $2.5 \mathrm{~kg} / \mathrm{d}$. Thus, the milk loss that could be associated with these disorders was insignificant.

\section{Reproductive Management Variables}

In model 2, inclusion of reproductive management variables that indirectly measure reproductive performance and act as disease prevention options elucidate the relationship between MPE and reproductive disorders. Replacement of cows that are predisposed to disorders prevents reoccurrence of those disorders in subsequent lactations. Similarly, reproductive disorders may be reduced if freshly enrolled cows are older. To explain the significant association between cow ages at first calving and MPE, the supplementary regression analysis, the Pearson correlation analysis, and the analysis comparing the mean of variables for low-, medium-, and high-efficiency score groups were evaluated.

The regression results suggest that a cow's age at first calving is a significant factor and is negatively associated with all the reproductive disorders except for dystocia. The replacement rate was significant and negatively associated with dystocia, retained placenta, and induction of birth. These results indicate that farms with a high replacement rate (i.e., a high number of cows at first calving), tend to experience fewer reproductive disorders whereas replacement rate and cow age at first calving are positively correlated: as 
age at calving increases, so does herd replacement rate. The correlation analysis also suggests that the reproductive disorder variables are all positively correlated with each other.

The comparison of reproductive disorder and management variables for different levels of MPE score groups is presented in Table 5 . The values indicate that farms with low MPE have, on average, higher cow age at first calving and higher replacement rates. The farms in this group may have a lower quantity of milk produced because cows calving for the first time in such herds tend to produce less milk than older cows, which in turn, results in lower MPE. This is not to say that higher age at first calving is associated with lower genetic improvement. The farms with high MPE scores (>95) and with the opposite characteristics tended to benefit from the genetic improvements associated with slightly shorter generation intervals because of younger ages at first calving. Farmers' knowledge of fresh cows having genetically high milk output capabilities increased the probability of using the fresh cows to replace older ones with minor reproductive disorders.

The issues of possible nonreporting bias are difficult to investigate. However, it does not seem to be a significant influence for this study. Bennedsgaard (2003) and Lawson (2003) have discussed the issue of nonreporting bias for disease treatment records.

\section{CONCLUSIONS}

With the caveats discussed in the previous section, it is concluded that reproductive disorders do not have a negative relationship with milk production efficiency on Danish dairy farms. This is due to compensating management actions, such as early enrollment and replacement of cows with disorders. However, the conclusion is contrary to the expected negative biological impact of the disorders. Dairy farms with MPE scores below $90 \%$ have room for improvement to become more efficient and to better cope with price fluctuations. They may therefore be subjects of focus and concern for agricultural advisors.

The dairy advisor can apply efficiency scores to identify inefficient herds. Advisors can use the results of stochastic frontier analysis, which investigates the effect of alternative management procedures on the individual herd's efficiency to advise farmers. However, in order to conduct future investigations of the effect of management changes, studies need to include evaluations of other production diseases. Development of efficiency analysis based on empirical data may also be considered as an alternative to simulation models based on specialists' belief networks and data from the literature.

The policy implications for these findings are as follows: It is essential to continue to ensure that incidence levels of reproductive disorders are kept at a level that does not increase the cost of production excessively. Cost is a major component of economic efficiency, which was not addressed herein. Milk production efficiency as measured in the current study may differ from optimal economic efficiency.

\section{ACKNOWLEDGMENTS}

The Danish Research Training Council and The Royal Veterinary and Agricultural University are acknowledged for financing the senior author's Ph.D. program in Animal Health Economics. R. Piggot and the staff of the School of Economics, University of New England (UNE), Armidale, Australia, are acknowledged for their kind hospitality during the first author's visit to UNE from October 2001 to April 2002. We thank G. Battese, UNE, for his useful comments on the earlier draft of this paper. We thank the 2 anonymous reviewers for their useful comments. All the comments have greatly helped to improve this paper.

\section{REFERENCES}

Aigner, D., C. A. K. Lovell, and P. Schmidt. 1977. Formulation and estimation of stochastic frontier production function models. J. Economet. 6:21-37.

Anonymous. 1996. Økonomien i landbrugets driftsgrene serie B nr 82 (Economics of Agricultural Enterprises). Danish Research Institute of Food Economics number B82, Copenhagen Denmark.

Anonymous. 1998. Økonomien i landbrugets driftsgrene serie nr 82 (Economics of Agricultural Enterprises). Danish Research Institute of Food Economics number B82, Copenhagen Denmark.

Battese, G. E., and T. J. Coelli. 1988. Prediction of firm-level technical efficiencies with a generalized frontier production function and panel data. J. Economet. 38:387-399.

Battese, G. E., and T. J. Coelli. 1995. A model for technical inefficiency effects in a stochastic frontier production function for panel data. Empir. Econ. 20:325-332.

Bartlett, P.C., J. H. Kirk, M. A.Wilke, J. B. Kaneene, and E. C. Mather. 1986. Metritis complex in Michigan Holstein-Friesian cattle: Incidence, descriptive epidemiology and estimated economic impact. Prev. Vet. Med. 4:235-248.

Beattie, B. R., and C. R. Taylor. 1985. The Economics of Production. John Wiley \& Sons, Inc., New York, NY.

Bennedsgaard, T. 2003. Reduced use of veterinary drugs in organic dairy herds-Potentials and consequences. Ph.D. Thesis. The Royal Veterinary and Agricultural Univ., Copenhagen, Denmark.

Bravo-Ureta, B. E., and L. Rieger. 1990. Alternative production frontier methodologies and dairy farm efficiency. J. Agric. Econ. 41:215-226.

Bravo-Ureta, B. E., and L. Rieger. 1991. Dairy farm efficiency measurement using stochastic frontier and neoclassical duality. Am. J. Agric. Econ. 73:421-428. 
Bruun J., A. K. Ersbøll, and L. Alban. 2002. Risk factors for metritis in Danish dairy cows. Prev. Vet. Med. 54:179-190.

Coelli, T. J. 1995. Recent developments in frontier modelling and efficiency measurement. Aust. J. Agric. Econ. 39:219-245.

Coelli, T. J. 1996. A guide to FRONTIER version 4.1: A computer program for frontier production and cost function estimation. Mimeo, CEPA Working Paper, No 96/07. Dept. Econometrics, Univ. of New England, Armidale, Australia.

Coelli, T. J., D. S. P. Rao, and G. E. Battese. 1998. An Introduction to Efficiency Studies and Productivity Analysis. Kluwer Academic Publishers, Boston, MA.

Cuesta, R. A. 2000. A production model with firm-specific temporal variation in technical inefficiency: With application to Spanish dairy farms. J. Prod. Anal. 13:139-158.

Debertin, D. L. 1986. Agricultural Production Economics. Macmillan Publishing Co. New York, NY.

Erb, H. N., S. W. Martin, N. Ison, and S. Swaminathan, 1981. Interrelationships between production and reproductive diseases in Holstein cows. Path analysis. J. Dairy Sci. 64:282-289.

Fourichon, C., H. Seegers, N. Bareille, and F. Beaudeau. 1999. Effects of disease on milk production in the dairy cow: A review. Prev. Vet. Med. 41:1-35.

Fourichon, C., H. Seegers, F. Beaudeau, L. Verfaille, and N. Bareille. 2001. Health-control costs in dairy farming systems in western France. Livest. Prod. Sci. 68:141-156.

Fourichon, C., H. Seegers, and X. Malher. 2000. Effects of disease on reproduction in the dairy cow: A meta-analysis. Theriogenology 53:1729-1759.

Gröhn, Y. T., H. N. Erb, C. E. Mcculloch, and H. S. Saloniemi. 1990. Epidemiology of reproductive disorders in dairy cattle: Associations among host characteristics, disease and production. Prev. Vet. Med. 8:25-39.

Hallam, D., and F. Machado. 1996. Efficiency analysis with panel data: A study of Portuguese dairy farms. Eur. Rev. Agric. Econ. 23:79-93.
Jondrow, J., C. A. K. Lovell, I. S. Materov, and P. Schmidt. 1982. On the estimation of technical inefficiency in the stochastic frontier production function model. J. Economet. 19:233-238.

Kodde, D. A., and F. C. Palm. 1986. Walds criteria for jointly testing equality and inequality restrictions. Econometrica 54:12431248.

Kumbhakar, S. C., B. Biswas, and D. V. Bailey. 1989. A study of economic efficiency of Utah dairy farmers: A system approach. Rev. Econ. Stat. 71:595-604.

Kumbhakar, S. C., S. Ghosh, and J. T. McGuckin. 1991. A generalized production frontier approach for estimating determinants of inefficiency in U. S. dairy farms. J. Business Econ. Stat. 9:279-286.

Lawson, L. G. 2003. Animal health economic: Efficiency and endemic disease control in Danish dairy herds. Ph.D. Diss. The Royal Veterinary and Agricultural University, Copenhagen, Denmark.

Lawson, L. G., K. E. Baptiste, M. Lund, and J. F. Agger. 2003. The relationship between technical inefficiency of milk production and udder disorders in Danish dairy cattle herds. Mimeo. Dept. and Animal Science and Animal Health, The Royal Veterinary and Agricultural University, Denmark.

Meeusen, W., and J. van den Broeck. 1977. Efficiency estimation from Cobb-Douglas production functions with composed error. Int. Econ. Rev. 18:435-444.

Nebel, R. L., and M. L. McGilliard. 1993. Interactions of high milk yield and reproductive performance in dairy cows. J. Dairy Sci. 76:3257-3268.

Nielsen, U. S., G. P. Aamand, and T. Mark. 2000. National genetic evaluation of udder health and other health traits in Denmark. Proc. 2000 Interbull Mtg. Bull. 25:143-150. Bled, Slovania.

Østergaard, S., and Y. T. Gröhn. 1999. Effects of disease on test day milk yield and body weight of dairy cows from Danish research herds. J. Dairy Sci. 82:1188-1201.

Rajala-Schultz, P. O., and Y.T.Gröhn. 1999. Culling of dairy cows. Part II. Effects of diseases and reproductive performance on culling in Finnish Ayrshire cows. Prev. Vet. Med. 41:279-294.

Tauer, L. W. 2001. Efficiency and competitiveness of the small New York dairy farm. J. Dairy Sci. 84:2573-2576. 